\title{
Combination of bulk laxative along with probiotics and prebiotics in the management of irritable bowel syndrome with constipation: A safe \& effective option
}

\author{
Arif Ahmed Faruqui', B Jamaiwar'2, B Sharma ${ }^{3}$ \\ ${ }^{1}$ M.D. Clinical Pharmacologist, 504 A, Rizvi Mahal, Waterfield Road, Bandra (West), Mumbai - 400050 Maharashtra, \\ ${ }^{2}$ General Surgeon, Nandavan Layout, Nagpur - 440 010, Maharashtra, ${ }^{3}$ Medical Officer, Chhindwara - 480001, \\ Madhya Pradesh, India
}

\section{A B S T R A C T}

Background: Irritable bowel syndrome (IBS) is a common and relapsing functional bowel disorder. This disease has a substantial global burden and is associated with significant disability and high costs that adversely affects the health related quality of life of large population worldwide. Aims and Objective: We studied the efficacy and tolerability of bulk laxative plus probiotic and prebiotic in the treatment of constipation associated IBS (IBS-C) in Indian patients. Materials and Methods: Eighty patients with symptoms of IBS-C were enrolled in a post-marketing surveillance, multicentre, open label, non-randomized, noncomparative study where EVAQ- P - combination of fiber (psylliun husk/lspaghula, probiotic and prebiotic) was given BID for 6 weeks. The symptoms were assessed at baseline, and after completion of 2, 4 and 6 weeks of therapy. Results: Results showed a significant trend ( $p<0.0001$ ) of improvement from week 2 for abdominal pain, abdominal fullness, defecation frequency, straining during defecation, constipation and subject global assessment of relief (SGA). Approximately $6 \%$ patients showed flatulence (mild) as a treatment emergent side effect. Conclusion: Combination of ispaghula along with a blend of probiotics and prebiotic twice daily for 6 weeks is efficacious, safe, and well-tolerated in IBS-C patients.

\section{Access this article online}

Website:

http://nepjol.info/index.php/AJMS DOI: 10.3126/ajms.v8i1.12909 E-ISSN: 2091-0576

P-ISSN: 2467-9100

Key words: Irritable bowel syndrome, Probiotics, Constipation, Prebiotics, Ispaghula

\section{INTRODUCTION}

Functional gastrointestinal diseases (FGID) are commonly encountered disturbances of gut function that are characterized by symptoms arising in the mid and lower gut. FGID has been a diagnostic problem without specific pathological or radiological markers and therapies targeted against them often being associated with poor efficacy and side effects.

Functional gastrointestinal disease has been defined into various subsets:

- Functional dyspepsia

- Epigastric pain syndrome
- Postprandial distress syndrome

- Cyclic vomiting syndrome

- Chronic idiopathic nausea

- Irritable bowel syndrome (IBS).

IBS is a common and relapsing functional bowel disorder. ${ }^{1}$ IBS also produces a significant economic burden due to both direct health care-related costs and indirect costs due to impaired work productivity. ${ }^{2}$ In India, the prevalence of IBS ranges from 4.2 to $7.9 \%$ with more men patients than females with predominance of upper abdominal symptoms. ${ }^{3}$

The severity of IBS ranges from mild, sporadic symptoms, to severe, invalidating symptoms. It has been shown that 
severity is closely related to the impact of the disease on patient's quality of life. ${ }^{3,4}$

As number of factors plays an important role in its etiology, the unifying cause of the disease is not entirely apparent and the disease is diagnosed by specific set of symptoms as decided by Rome criteria. However, definitive diagnosis of IBS presents challenges to the gastroenterologists due to overlap in symptom presentations with other diseases or associated conditions. ${ }^{5}$

Although the pathogenesis of IBS is not known, a multifactorial involvement of diet, gene mutations, psychosocial factors, and immune mediated processes are hypothesized. The contribution of these factors varies and in many cases no single cause can be determined. ${ }^{6}$ Currently available IBS therapies are mainly symptom orientated (e.g., antidiarrheals for diarrhea, laxatives for constipation or smooth muscle relaxants for pain) and often are of limited efficacy regarding the overall complaints.

In the last few years, increasing evidence for the role of gut bacteria in the control of gut function has been recognized, and recent studies using novel techniques for the quantification of gut microflora have demonstrated differences in the flora of patients with IBS compared to healthy subjects. Supplementation of probiotic bacteria may have beneficial effects in IBS symptoms. ${ }^{7,8}$ Studies with combination of fiber, prebiotic \& probiotic has been published earlier proving the efficacy and safety of this combination in the management of IBS symptoms in western population. This study was conducted to evaluate efficacy and safety with the use of this combination in Indian patients suffering from IBS-C.

\section{MATERIALS AND METHODS}

In this study, we evaluated the effect of probiotics in combination with dietary fiber and prebiotic on the symptoms of IBS. This was a post-marketing surveillance, multicentre, open label, non-randomized, non-comparative trial in Indian patients.

In this study, a fixed dose combination of Ispagbula Husk 3.5 gm, L. acidophilus 1500 Millions, L. plantarum 500 Millions, Bifidobacterium longum 500 Millions Bifidobacterium Infantis 500 Millions, Fructooligosaccharides 50 mg; (Brand name: EVAQ-P; Medley Pharmaceuticals Ltd. Mumbai, India) was administered to 80 patients suffering from IBS symptoms twice a day.

\section{Inclusion criteria}

Patients of either gender who were willing to give informed consent were eligible to be included in the study if they had a clinically confirmed diagnosis of IBS as per Rome III criteria.

\section{Exclusion criteria}

Patients were excluded from the study if they had a known/ suspected history of hypersensitivity to any of the EVAQ-P active ingredients or patients suffering from lactose intolerance. Furthermore, patients suffering from intestinal obstruction or patients with immuno- compromised state were also excluded from the study.

\section{Treatment}

Patients participating in this study were prescribed with EVAQ-P orally 2 Spoons (10 gm) BID (bis in die: Twice a day) for 42 days (6 weeks). (2 teaspoonful of EVAQ $P$ powder to a glass of water, stir well and consume the content immediately. Follow it up with a glass of water).

The study comprised of 4 visits in total; Baseline visit in which patients were screened and enrolled in the study. From day 1 of the study, treatment was initiated. Visit 1 and 2 were scheduled after 2 and 4 weeks of the initiation of treatment respectively to assess and record the patient compliance and symptom control during the treatment. These visits were followed by the final visit 3 , after completion of 6 weeks therapy to record the final study outcome.

\section{Assessment of outcome}

The symptoms were assessed before initiation of the therapy (as a baseline visit), after completion of 2, 4 and 6 weeks of the therapy. The symptoms evaluated were: abdominal fullness, abdominal pain, defecation frequency, straining during defecation, constipation followed by subject global assessment of relief (SGA).

Numeric rating scale (NRS) for IBS trial using a scale from 0 (none) to 10 (worst possible pain) to describe the worst abdominal pain episode from the past $24 \mathrm{hrs}$ was used. Straining during defecation was evaluated using 5 point scale $(+2=$ significantly relieved, $+1=$ moderately relieved, $0=$ unchanged, $-1=$ moderately worse, and $-2=$ significantly worse). SGA was also expressed and recorded in Likert 5 point scale $(1$ - not at all, 2 - slightly, 3 - moderately, 4 - quite a bit, 5 - extremely). Another Likert scale ( +2 to -2$)$ was also used to evaluate constipation $(+2=$ significantly relieved, $+1=$ moderately relieved, $0=$ unchanged, $-1=$ moderately Worse, and -2 significantly worse for constipation).

\section{Adverse event monitoring}

An adverse event was defined as any untoward medical occurrence in a patient, regardless of whether it was 
considered to be causally related to use of the treatment. Adverse events data were collected throughout the study and until 30 days after the last dose. Information recorded concerning the adverse events occurring during the trial period included their nature, whether serious or not, their intensity, any treatment given, the outcome, and an opinion about the causal relationship with the study medication.

\section{Statistical analysis}

Data analysis on patient demographics and various outcome measures were performed using graph pad prism. ${ }^{6}$ Comparison between the baseline values with the value on the week 2, week 4 and week 6 of the treatment were made, as well as comparison in between these days by applying 1 way analysis of variance (ANOVA) \& the post hoc Turkeys multiple comparison test. Values of $\mathrm{P}<0.05$ were considered significant.

\section{RESULTS}

\section{Patient distribution}

Investigators at 08 centers recruited the patients and the trial was conducted from June 2014 to December 2014. A total of 80 patients were enrolled (Table 1: Subject demographics) and received the dose of study drug. Overall, at baseline $77 \%$ patients were suffering for constipation as a primary IBS symptom.

\section{Efficacy}

All the efficacy parameters showed a significant trend of improvement from week 2 , however at week 4 , all of the baseline symptoms such as flatulence, frequency of defecation, and stool consistency significantly improved compared to the baseline (Figures 1-6). However, the changes in the frequency of bowel movement and abdominal fullness were significantly higher $(p<0.0001$; Table 2).

\section{Subject global assessment of relief}

Subject global assessment, which was measured using a Likert 5 point scale; like; 1 not at all, 2 slightly, 3 moderately, 4 quite a bit, 5 extreme reliefs, showed overall improvement in the baseline symptoms. At week 3; 30\% patients showed grade 4 reliefs compared to baseline. Furthermore, at the end of the study, nearly $44 \%$ patients were rated to had extreme relief (grade 5) and 36\% of

\begin{tabular}{lc} 
Table 1: Subject demographics \\
\hline \multicolumn{2}{c}{ Baseline characteristics of all patients } \\
\hline Male/female $(\mathrm{n})$ & $53 / 27$ \\
Age (yrs) range & $21-65$ \\
\hline
\end{tabular}

patients showed quite a bit relief (grade 4) from the baseline symptoms (Table 2). This suggests, nearly $80 \%$

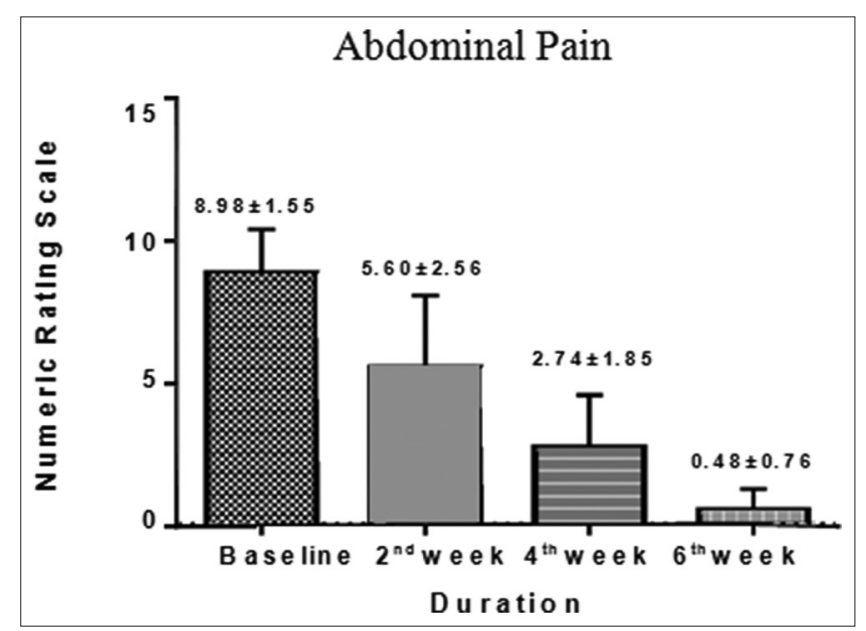

Figure 1: Change in the severity of abdominal pain from baseline to week $6(p<0.0001)$

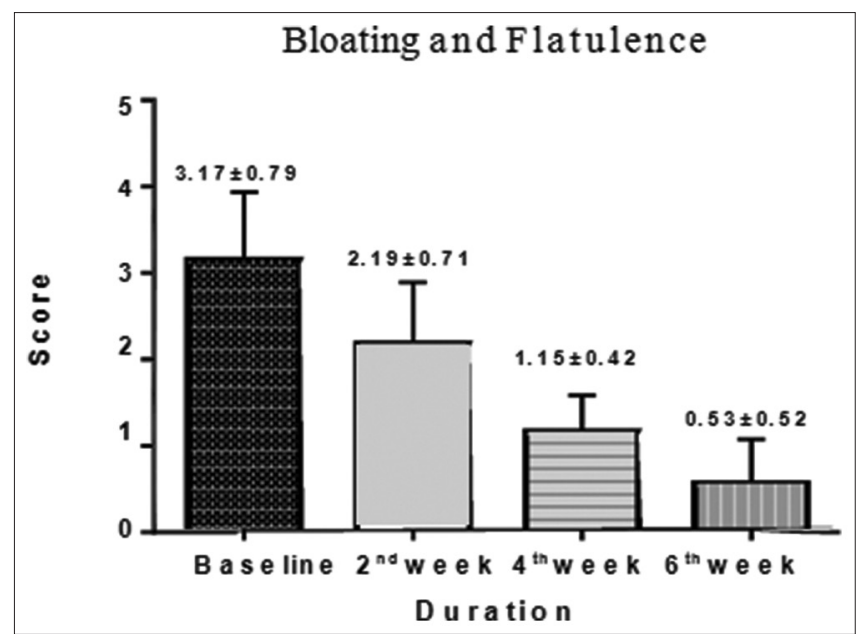

Figure 2: Change in the severity of abdominal fullness from baseline to week $6(p<0.0001)$

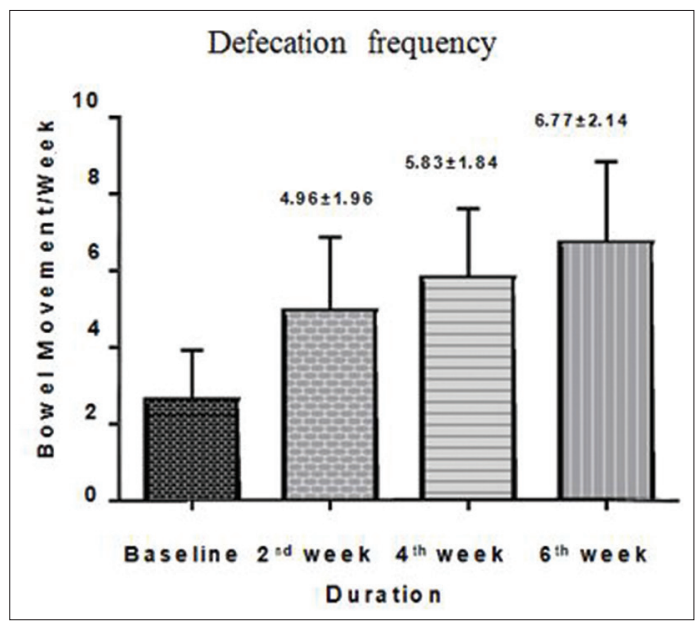

Figure 3: Change in the severity of defecation frequency from baseline to week $6(p<0.0001)$ 


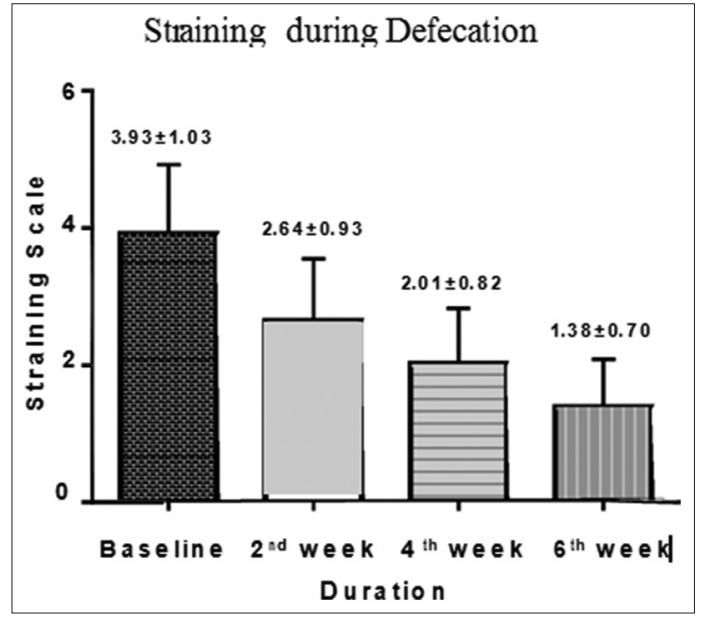

Figure 4: Change in the severity of straining during defecation from baseline to week $6(p<0.0001)$

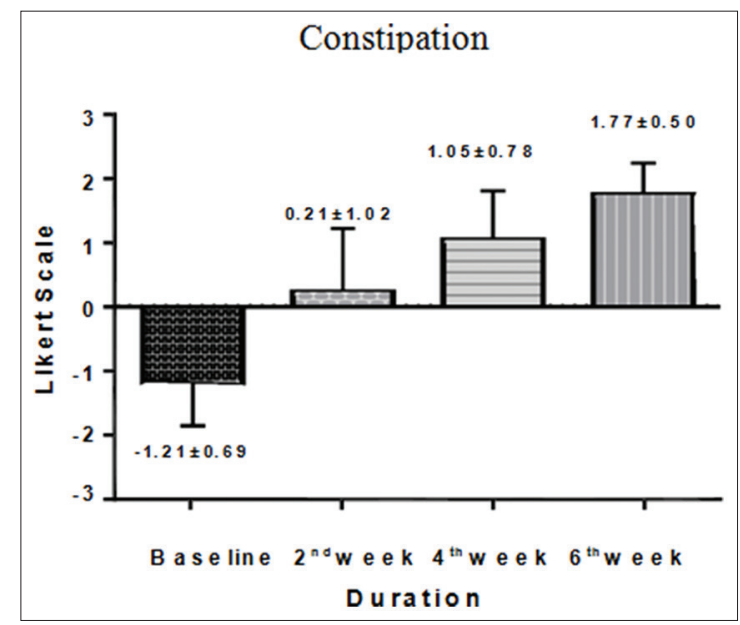

Figure 5: Relief from constipation $(p<0.0001)$

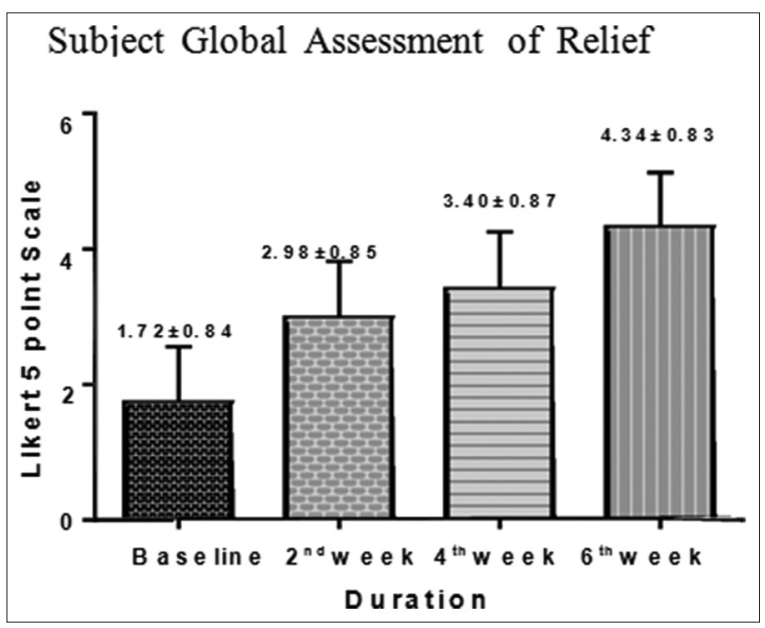

Figure 6: Subject global assessment $(p<0.0001)$

patients rated the efficacy of global symptom assessment as grade 4 or above, suggesting a significant improvement in overall relief (Figure 6).

\section{Safety and tolerability}

Treatment-emergent adverse events (AE) were experienced by 5 patients $(\sim 6 \%)$ who received EVAQ-P. These patients primarily reported flatulence as a side effect. Most of these AEs were mild or moderate in severity $\&$ resolved on its own. None of the patient died or prematurely discontinued during the study.

\section{DISCUSSION}

Irritable bowel syndrome (IBS) is a common disorder characterized by abdominal pain and altered bowel habit for at least 3 months. It is a complex, heterogeneous condition of unknown origin, with a variety of different factors involved in symptom generation. These include: increased visceral sensitivity, altered motility and gas transport, low-grade inflammation, psychological disturbances and early life experiences. ${ }^{9}$ The goal of treatment for IBS is improving the patient's quality of life by decreasing global IBS symptoms with non- pharmacologic and pharmacologic treatments. ${ }^{10}$

Increased dietary fiber is frequently recommended for irritable bowel syndrome. Improvement in IBS symptoms seems to be due to main mechanism of action of fiber that stimulates oro-anal transit and slow the intra-colon pressures. ${ }^{11}$ Probiotics are responsible for strengthening and increasing the gut barrier function, hindering pathogen binding and regulating the gut inflammatory response. Furthermore, it was also observed that, use of probiotics simultaneously reduces the visceral hypersensitivity associated with both inflammation and psychological stress. $^{12-14}$

Several clinical studies have demonstrated the efficacy of probiotics for the treatment of patients with IBS. The most commonly studied probiotics generally includes Lactobacillus, Bifidobacteria, and Streptococii. Agrawal et al. reported that it was necessary to select an organism or a mixture of probiotics that exhibit the properties most suitable for the particular expected health benefit or physiological effect. ${ }^{15}$ In a study by Choi SC, et al. it was observed that probiotic fermented milk containing dietary fiber was more effective particularly in those patients with the constipation-predominant IBS. ${ }^{16}$ The said study confirmed the available evidence that fiber appears to play a role in the management of patients with IBS, especially if constipation is the primary concern. Although insoluble fiber is not better than placebo but soluble fiber in particular showed favourable results in IBS related constipation. ${ }^{17} \mathrm{Kim}$ et al. in another study suggested that effect of fiber and probiotics was different among the given organisms and IBS subtypes. ${ }^{18}$ The present study 


\begin{tabular}{|c|c|c|c|c|c|c|}
\hline \multirow[t]{2}{*}{ Sr. No. } & \multirow[t]{2}{*}{ Parameters } & \multicolumn{4}{|c|}{ Mean $\pm S D$} & \multirow[t]{2}{*}{$P$ value } \\
\hline & & Baseline (BL) & Week 2 (W-2) & Week 4 (W-4) & Week 6 (W-6) & \\
\hline 1 & Constipation & $-1.21 \pm 0.69$ & $0.21 \pm 1.02$ & $1.05 \pm 0.78$ & $1.77 \pm 0.50$ & $\begin{array}{c}\text { BL vs. W-2, W-4, W-6: } \\
\quad P<0.0001\end{array}$ \\
\hline 2 & Abdominal pain & $8.98 \pm 1.55$ & $5.60 \pm 2.56$ & $2.74 \pm 1.85$ & $0.48 \pm 0.76$ & $\begin{array}{c}\text { BL vs. W-2, W-4, W-6: } \\
\quad P<0.0001\end{array}$ \\
\hline 3 & Abdominal fullness & $3.17 \pm 0.79$ & $2.19 \pm 0.71$ & $1.15 \pm 0.42$ & $0.53 \pm 0.52$ & $\begin{array}{c}\text { BL vs. W-2, W-4, W-6: } \\
\quad P<0.0001\end{array}$ \\
\hline 4 & Straining during defecation & $3.93 \pm 1.03$ & $2.64 \pm 0.93$ & $2.01 \pm 0.82$ & $1.38 \pm 0.70$ & 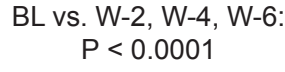 \\
\hline 5 & Defecation frequency/week & $2.62 \pm 1.32$ & $4.96 \pm 1.96$ & $5.83 \pm 1.84$ & $6.77 \pm 2.14$ & $\begin{array}{c}\text { BL vs. W-2, W-4, W-6: } \\
P<0.0001 \\
\text { W-4 vs. W-6: } \\
\text { P }<0.01\end{array}$ \\
\hline 6 & $\begin{array}{l}\text { Subject global assessment } \\
\text { of relief }\end{array}$ & $1.72 \pm 0.84$ & $2.98 \pm 0.85$ & $3.40 \pm 0.87$ & $4.34 \pm 0.83$ & $\begin{array}{c}\text { BL vs. W-2, W-4, W-6: } \\
\text { P }<0.0001 \\
\text { W-2 vs. W-4: } \\
\text { P }<0.01\end{array}$ \\
\hline
\end{tabular}

thus incorporated the use of soluble fiber ispaghula along with probiotics and prebiotics, to get favorable results in the relief of symptoms of constipation.

World Health Organisation considers probiotics as live microorganisms which when administered in adequate doses; confer health benefits to the host. ${ }^{19}$ Rafter et al. have suggested several mechanisms of action, that includes: stimulation of the host immune response (by increasing the phagocytic activity, IgA production and $\mathrm{T}$ and $\mathrm{B}$ lymphocytes activation); binding and degradation of compounds with carcinogenic potential; qualitative and/or quantitative changes in the intestinal microbiota involved in the production of carcinogens; antimutagenic producing compounds in the colon (like butyrate); change in the metabolic activity of the intestinal microbiota; alteration of physicochemical conditions of the colon with decreasing $\mathrm{pH}$; and effects on the host physiology. ${ }^{20}$

Combining both a probiotic and a prebiotic in a single food product provides the following advantages, as stated in the synbiotic definition, an improved survival during the passage of the probiotic bacteria through the upper intestinal tract and a more efficient implantation in the colonic microbiota together with a stimulating effect of the prebiotic on the growth and/or the activities of both the exogenous (probiotic) and endogenous bacteria (e.g., bifidobacteria). ${ }^{21}$ Results of the present study are comparable to the previous studies. With the use of probiotics along with prebiotics and ispaghula, the present study showed significant improvement in the IBS symptoms mainly constipation, abdominal fullness and abdominal pain at the end of 6 weeks.

In this prospective, multicentric, observational study we found that combination of Ispaghula along with a probiotic and prebiotic twice daily for 6 weeks is effective, safe, and well-tolerated in IBS-C patients. Furthermore, patients experienced a significant relief in IBS symptoms of constipation, abdominal pain, fullness and strain during defecation, and this symptomatic relief was obtained during the first 2 weeks of treatment and sustained during the following 4 weeks.

\section{ACKNOWLEDGEMENT}

Authors acknowledge the immense help received from the scholars whose articles are cited and included in references of this manuscript. The authors are also grateful to authors/editors/publishers of all those articles, journals and books from where the literature for this article has been reviewed and discussed. Authors also acknowledge the support of all the following doctors for providing their observations. Writing support (including statistical analysis and tabulating the data) was provided by Mr. Shailesh Pallewar and Mr. Altamash Momin.

\section{Competing interests}

All authors had access to the data and vouch for the veracity and completeness of the data and the data analysis. No other author reported any conflict of interest.

\section{REFERENCES}

1. World Gastroenterology Organization. World Gastroenterology Organization Global Guideline: Irritable bowel syndrome: Aglobal perspective-April 20, 2009. South African Gastroenterology Review, 7(3), 23-30.

2. Vahedi H, Ansari R, Mir-Nasseri MM, and Jafari E. Irritable Bowel Syndrome: A Review Article. Middle East J Dig Dis Sep 2010; 2(2): 66-77.

3. Association of Physicians of India. Irritable Bowel Syndrome: 
The Indian Scenario; Medicine Update 2013.

4. Coffin B, Dapoigny M, Cloarec D, Comet D and Dyard F. Relationship between severity of symptoms and quality of life in 858 patients with irritable bowel syndrome. Gastroenterol Clin Biol 2004; 28: 11-15.

5. Saunjoo Y, Oliver G, Laura K and Lana F. Management of Irritable Bowel Syndrome (IBS) in Adults. Altern Med Rev 2011; $16(2): 134-151$.

6. Mathew $P$ and Bhatia SJ. Pathogenesis and management of irritable bowel syndrome. Trop Gastroenterol 2009; 30(1): 19-25.

7. Rajilic-SM, Biagi E, Heilig HG, Kajander K, Kekkonen RA, Tims S, et al. Global and deep molecular analysis of microbiota signatures in fecal samples from patients with irritable bowel syndrome. Gastroenterology 2011; 141(5): 1792-1801.

8. Moayyedi P, Ford AC, Talley NJ, Cremonini F, Foxx-Orenstein AE, Brandt LJ, et al. The efficacy of probiotics in the treatment of irritable bowel syndrome: a systematic review. Gut 2010; 59(3): 325-332.

9. Elizabeth AW, XuiLi N and Bernard MC. Dietary intakes in people with irritable bowel syndrome.BMC Gastroenterol 2011; 11(1):9.

10. Lekha S. Irritable bowel syndrome: Pathogenesis, diagnosis, treatment, and evidence- based medicine. World J Gastroenterol 2014; 20(22): 6759-6773.

11. Viera AJ, Hoag S and Shaughnessy J. Management of Irritable Bowel Syndrome. Am Fam Physician 2002; 66(10): 1867-1874.

12. Camilleri M. Management of the irritable bowel syndrome. Gastroenterol 2001; 120(3): 652-668.

13. Camilleri M, Heading RC and Thompson WG. Clinical perspectives, mechanisms, diagnosis and management of irritable bowel syndrome. Aliment Pharmacol Ther 2002; 16(8): 1407-1430.
14. Zuckerman MJ. The role of fiber in the treatment of irritable bowel syndrome: therapeutic recommendations. J Clin Gastroenterol 2006; 40(2): 104-108.

15. Agrawal A, Houghton LA, Morris J, Reilly B, Guyonnet D, Goupil Feuillerat $\mathrm{N}$, et al. Clinical trial: the effects of a fermented milk product containing Bifidobacterium lactis $\mathrm{DN}-173-010$ on abdominal distension and gastrointestinal transit in irritable bowel syndrome with constipation. Aliment Pharmacol Ther 2009; 29(1): 104-114.

16. Choi SC, Kim BJ, Rhee PL, Chang DK, Son HJ, Kim JJ, et al. Probiotic Fermented Milk Containing Dietary Fiber Has Additive Effects in IBS with Constipation Compared to Plain Probiotic Fermented Milk. Gut Liver 2011; 5(1): 22-28.

17. Bijkerk CJ, Muris JW, Knottnerus JA, Hoes AW and de Wit NJ. Systematic review: the role of different types of fibre in the treatment of irritable bowel syndrome. Aliment Pharmacol Ther 2004; 19(3): 245-251.

18. Kim HJ, Vazquez Roque MI, Camilleri M, Stephens D, Burton DD, et al. A randomized controlled trial of a probiotic combination VSL\# 3 and placebo in irritable bowel syndrome with bloating Neurogastroenterol Motil 2005; 17(5): 687-696.

19. Institute of Medicine (US) Forum on Microbial Threats. Ending the War Metaphor: The Changing Agenda for Unraveling the Host-Microbe Relationship: Workshop Summary. Washington (DC): National Academies Press (US); 2006. Summary and Assessment. Available from: https://www.ncbi.nlm.nih.gov/ books/NBK57060.

20. Rafter J. Probiotics and colon cancer. Best Pract Res Clin Gastroenterol 2003; 17(5): 849-859.

21. Roberfroid MB. Prebiotics and synbiotics: concepts and nutritional properties. Br J Nutr 1998; 80(4): Suppl. 2, S197-S202.

\section{Authors Contribution:}

AAF - Concept and design of the study, reviewed the literature, manuscript preparation and critical revision of the manuscript; BJ - Providing the patients data in required case record forms; BS - Providing the patients data in required case record forms.

Source of Support: Nil, Conflict of Interest: None declared. 